\title{
REFLEXIONES ACERCA DE UN PROGRAMA DE ELE: DESDE LA PERSPECTIVA DE LOS ESTUDIANTES
}

\author{
REFLECTIONS ABOUT AN ELE PROGRAM: \\ FROM THE STUDENTS' PERSPECTIVE \\ REFLEXÕES SOBRE UM PROGRAMA ELE: \\ DO PONTO DE VISTA DOS ESTUDANTES
}

\section{Numas Armando Gil Olivera' ${ }^{1}$ \\ Dayson David Ahumada Ebratt ${ }^{2}$}

Universidad Del Atlántico

Universidad Del Norte

\section{RESUMEN}

El presente artículo de reflexión tiene como objetivo describir las perspectivas de los estudiantes de un programa de ELE llamado Didáctica de la enseñanza del español como 1 Orcid: 0000-0002-8991-4348

Dirección: Calle 44 \#26-13, Barranquilla, Colombia.

Correo electrónico: ngilolivera@mail.uniatlantico.edu.co

Títulos académicos: Magíster en Educación con énfasis en Educación Superior

Afiliación institucional: Docente tiempo completo ocasional en el departamento de Lenguas Extranjeras en la Universidad del Atlántico, Colombia.

Datos de la Universidad del Atlántico: ubicada en kilómetro 5, vía Puerto Colombia, Teléfono: (035) 3853002

\section{2}

Orcid: 0000-0002-5795-9293

Dirección: Calle 76 \# 32- 346 las mercedes P2. APT1.

Correo electrónico: ebrattd@uninorte.edu.co

Títulos académicos: Lic. En Idiomas. Magíster en Educación con énfasis en Educación Superior

Afiliación institucional: Docente catedrático en la Universidad del Norte

Datos de la Universidad del Norte: ubicada en kilómetro 5, vía Puerto Colombia.

Teléfono: (035) 3509509 lengua extranjera perteneciente a la carrera de Licenciatura en Idiomas Extranjeros de la Universidad delAtlántico, Barranquilla, Colombia. Haciendo especial énfasis, en el alcance de los objetivos de aprendizaje que se plantea tal asignatura. En este sentido, esta investigación documental, descriptiva de tipo cualitativa, se centró en la revisión de los resultados de una encuesta llevada a cabo en el semestre 20203 y 2021-1 aplicada a los estudiantes de dos cursos que se enfocó en el logro de los objetivos de aprendizaje de los estudiantes. Para ello, sustentó los resultados en los distintos aportes teóricos de entidades como Marco Común Europeo, entre otros. $Y$ entre sus resultados reportó que la gran mayoría de los estudiantes si lograba alcanzar los objetivos de aprendizaje porque mostraban un amplio manejo de aspectos como los métodos y enfoques que se promovían 
en la asignatura. Este tipo de resultados, si bien no son absolutamente generalizables, si son un excelente punto de inicio para aquellos docentes de ELE que quieran incursionar y profundizar en estás temáticas.

\section{PALABRAS CLAVES:}

Lengua extranjera, Educación, Pedagogía, Aprendizaje

\section{REFLECTIONS ABOUT AN ELE PROGRAM: FROM THE STUDENTS' PERSPECTIVE}

\section{SUMMARY}

The purpose of this reflection article is to describe the perspectives of the students of an ELE program called Didactics of the Teaching of Spanish as a Foreign Language belonging to the Bachelor's Degree in Foreign Languages at the Universidad del Atlántico, Barranquilla, Colombia. Special emphasis was placed on the scope of the learning objectives of the course.

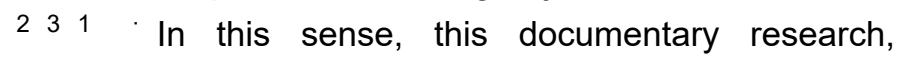
descriptive of qualitative type, focused on the review of the results of a survey carried out in the semester 2020-3 and 2021-1 applied to the students of two courses that focused on the achievement of the learning objectives of the students. For this purpose, it based the results on the different theoretical contributions of entities such as the Common European Framework, among others. And among its results, it reported that the vast majority of students did achieve the learning objectives because they showed a broad management of aspects such as the methods and approaches promoted in the subject. This type of results, although not absolutely generalizable, are an excellent starting point for those ELE teachers who want to dabble and go deeper into these topics.

\section{KEY WORDS}

Foreign language, Education, Pedagogy, Learning.

\section{REFLEXÕES SOBRE UM PROGRAMA ELE: DO PONTO DE VISTA DOS ESTUDANTES}

\section{ABSTRATO}

Este artigo visa descrever as perspectivas dos alunos de um programa ELE chamado Didática do ensino do espanhol como língua estrangeira pertencente ao diploma em Línguas Estrangeiras da Universidad del Atlántico, Barranquilla, Colômbia. A ênfase principal é colocada no escopo dos objetivos de aprendizagem deste assunto. Neste sentido, esta pesquisa documental, descritiva de tipo qualitativo, focalizou a revisão dos resultados de uma pesquisa realizada no semestre de 20203 e 2021-1 aplicada aos alunos de dois cursos que focalizaram a realização dos objetivos de aprendizagem dos alunos. Para isso, apoiou os resultados nas diversas contribuições teóricas de entidades como a Common European Framework, entre outras. E entre seus resultados relatou que a grande maioria dos estudantes foi capaz de alcançar os objetivos de aprendizagem porque mostraram uma ampla gestão de aspectos como métodos e abordagens que foram promovidos no assunto. Este tipo de resultados, embora não absolutamente generalizáveis, são um excelente ponto de partida para os professores da ELE que querem ir mais fundo nestes tópicos.

\section{PALAVRAS-CHAVE:}

Língua estrangeira, Educação, Pedagogia, Aprendizagem

\section{INTRODUCCIÓN}

A lo largo de la historia de la humanidad todas las disciplinas y áreas del saber han evolucionado a la par de la ciencia y la tecnología. Pero pocas han tenido el impacto que tiene la educación en la evolución de la sociedad. Puede afirmarse sin ninguna duda que el sistema educativo es el principal musculo del desarrollo y progreso de la sociedad humana. En la medida que una 
civilización tenga un buen sistema educativo su crecimiento y evolución será sostenible y prolongado.

En este sentido, dentro del sistema educativo existe un área llamada Lenguas Extranjeras o también conocida como Lenguas Modernas (según el país y región) la cual es manejada por docentes de idiomas se centran en la enseñanza de lenguas a estudiantes que van desde la educación inicial hasta la educación universitaria. Para fines de este estudio, se centrará en una asignatura electiva llamada Didáctica de la enseñanza del español como lengua extranjera que se ofrece a estudiantes de la carrera de Licenciatura en Idiomas Extranjeros de la Universidad del Atlántico, Barranquilla, Colombia.

La creación de esta asignatura se justificó de la siguiente forma:

Esta electiva nace de la experiencia de los

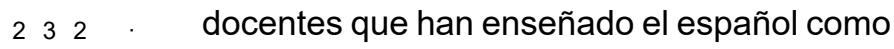
lengua extranjera en Colombia como fuera de ella, notamos que, en la licenciatura de lenguas extranjeras, nuestros estudiantes se gradúan con un muy buen nivel de lengua en inglés, francés y alemán (...) La idea de este seminario es formar docentes competentes en el campo de ELE con conocimientos actualizados, que puedan enfrentarse a este tipo de situaciones sin ningún inconveniente

Asimismo, esta asignatura contempla un macro objetivo general que reza lo siguiente:

- Proporcionar herramientas metodológicas y pedagógicas para la enseñanza del español como lengua extranjera

Para lograr tal propósito, se diseñaron varios objetivos específicos de aprendizaje que deben alcanzar los estudiantes como:
- Conocer los diferentes enfoques metodológicos en la de una lengua extranjera

- Familiarizarse con el marco común europeo de referencia en el contexto educativo

- Reconocer los niveles comunes de referencias

- $\quad$ Analizar técnicas y herramientas para utilizar de forma correcta las 4 destrezas

- Colocar en práctica los conocimientos adquiridos en el desarrollo de una clase ELE

- Conocer los diferentes aspectos de evaluación por destrezas.

En otros términos, el propósito de esta materia es capacitar a los docentes de ELE en los distintos enfoques, métodos, técnicas y estrategias en pro de la enseñanza- aprendizaje del español como lengua extranjera. Y para ello, contempla en su parcelación aspectos que van desde los niveles de manejo de una lengua extranjera contemplados por Marco Común Europeo de Referencia hasta los aspectos didácticos y pedagógicos que se han desprendido de los diferentes enfoques y métodos de enseñanza de una segunda lengua.

En este orden, el objetivo de este trabajo es determinar desde la perspectiva de los estudiantes si los objetivos del curso han sido alcanzados. Para ello, se elaboró una encuesta en donde se contemplan los aspectos más relevantes de los saberes que se estipulan en la parcelación y se fue respondida por 90 de los 150 estudiantes que hasta ahora han visto el curso. A continuación, se muestran los resultados junto a la sustentación teórica que conlleva este ítem de aprendizaje. 


\section{ENFOQUES METODOLÓGICOS MÁS UTILIZADOS EN LA ENSEÑANZA DE ESPAÑOL COMO LENGUA EXTRANJERA}

Dentro de la enseñanza de lenguas extranjeras los métodos y enfoques son minuciosamente elegidos con el fin de agilizar el aprendizaje de los estudiantes, en este sentido Martí (2017) explica que es importante que los docentes entiendan que el método abarca al enfoque, evidencia de ello es cuando afirma que "El método es el conjunto de procedimientos, contenidos $y$ técnicas empleados en el aula. Para definirlo, necesitamos estudiar los conceptos de enfoque, diseño y procedimiento. Por lo tanto, método es un hiperónimo de enfoque" (p. 22)

Dentro de la encuesta se les preguntó a los estudiantes acerca de temas como los enfoques más adecuados para enseñar el español como lengua extranjera, ítem que es ampliamente abordado a lo largo de la asignatura que fue descrita en el anterior segmento, en si la pregunta fue: ¿Cuáles son los enfoques más apropiados para enseñar español como lengua extranjera? $\mathrm{Y}$ estos fueron los resultados:

\section{A-Enfoque gramatical-traducción (5.3\%)}

\section{B-Enfoque estructural (5.5\%) \\ C-Enfoque comunicativo (36.6\%) \\ D-Enfoque por tareas $(52,6 \%)$}

Es evidente que la una mayoría de los estudiantes se inclina por el enfoque Comunicativo como el más adecuado para enseñar español como lengua extranjera. Es importante determinar de qué tratan estos enfoques para entender la respuesta de los estudiantes

En primer lugar, el enfoque gramaticaltraducción según autores como Gil (2019) tenía una "metodología que promovía el aprendizaje memorístico de normas morfosintácticas aplicándolas posteriormente, a textos escritos dejando de lado así, a la expresión oral" ( $p$. 173). Asimismo, el autor agrega: este enfoque tenía serias debilidades en su método como por ejemplo que las tareas como realizar listas de palabras o vocabulario, se traducían sin un entendimiento claro del contexto en el cual podían emplearse, asimismo las actividades eran sumamente limitadas en cuanto a la pedagogía y didáctica se refería (p. 173)

Es evidente que el enfoque gramatical traducción tenía vacíos en su metodología, pero se puede afirmar que gracias a sus aportes en la actualidad se toma en cuenta el vocabulario y las reglas gramaticales como elementos vitales en el desarrollo de competencias comunicativas en el aprendizaje de una segunda lengua. De ahí precisamente, se desprende la importancia de que los futuros docentes de español como lengua extranjera conozcan y entiendan los aportes de enfoques como el que se acaba de explicar.

Por su parte, el Enfoque Estructural según Gil (2019) "Surgió en el siglo XX y aunque se utilizó principalmente para la enseñanza del inglés como lengua extranjera después de la segunda guerra mundial, posteriormente se empleó para la enseñanza de otros idiomas como el español" (p. 173). Asimismo, Ortiz (2014) explica que se basó "en las teorías lingüísticas del estructuralismo de corte conductista, es decir, en las hipótesis de que las lenguas son un sistema de estructuras que pueden aprenderse a través de conductas, repetición." (p. 10).

Es evidente que guardaba una estrecha relación con el enfoque gramatical - traducción. Salvo que tenía premisas distintas en cuanto a la didáctica porque se enfocaba en la oralidad específicamente en la pronunciación correcta. Igualmente, partía de que el papel del docente era protagónico en el proceso de enseñanza - aprendizaje y que el rol del estudiante debía ser visto como pasivo, puesto que solo debía asimilar y repetir todos los lineamientos que le ofrecía el docente. 
Además, ha sido ampliamente criticado porque dentro de sus actividades solo se trabajan como textos ideales que distan muchísimo del uso real que los hablantes hacen del idioma en su cotidianidad (Gil (2019), Martínez (2009), Rall (2015), Pinto y Vargas (2015), Rojas (2019), Cervantes (2019), entre otros).

En tercer lugar, está el Enfoque comunicativo que el Centro Virtual de Cervantes (2017) señala como el enfoque que fusiona muchos de los aportes teóricos de los enfoques y métodos que lo antecedieron y los emplea en el desarrollo integral de las competencias comunicativas en la adquisición de la lengua extranjera, esto es evidente en el siguiente fragmento:

se conoce también como enseñanza comunicativa de la lengua (en inglés, Communicative Language Teaching), como enfoque nocional-funcional (en inglés, Notional-functional Approach) o como enfoque funcional (en inglés, Functional que con este modelo didáctico se pretende capacitar al aprendiente para una comunicación real -no sólo en la vertiente oral, sino también en la escrita- con otros hablantes de la LE; con este propósito, en el proceso instructivo a menudo se emplean textos, grabaciones y materiales auténticos y se realizan actividades que procuran imitar con fidelidad la realidad de fuera del aula

Es posible notar que el enfoque comunicativo se distingue de los demás enfoques porque parte de la noción de comunicación real. Es decir, enfoca sus actividades en situaciones que si se conectan con la realidad del habla de la lengua extranjera. Promoviendo así, una dinámica de interacción genuina entre los estudiantes que emule la realidad en la que tendrán que desenvolverse cuando tengan la oportunidad de hablar con hablantes nativos de la segunda lengua, esta perspectiva es apoyada por autores como Molina \& Ferreira (2008), Zayas (2014),

Casero (2016), Pastran, Et all (2020), entre otros.

En cuarto lugar, está el Enfoque por tareas que es considerado según Gil (2019) como "el heredero del enfoque comunicativo, porque parte de los componentes gramaticales, discursivos, sociolingüísticos y pragmáticos de la comunicación" (p. 174). Pero, se distingue del anterior porque se basa en la realización de pequeñas actividades consideradas como tareas. De ahí se desprende precisamente la definición que hacen Richards y Rodgers, citados en Gil (2019) "como aquel cuya metodología se basa en la realización de tareas como principal unidad de planeación e instrucción en el proceso de enseñanza de LE que promueve, además, el fortalecimiento de habilidades y destrezas comunicativas" (p. 176).

En este orden de las ideas, el hecho de que los estudiantes hayan elegido estos dos últimos enfoques en sus opciones de respuestas, permite afirmar que ellos si alcanzaron un nivel de entendimiento apropiado de la importancia de usar ciertos enfoques en la enseñanza del español como lengua extranjera. Esta concepción de que el enfoque comunicativo y el enfoque por tareas son los más completos en la enseñanza de lenguas extranjeras es compartida por muchos autores como Cortes (2000), Martínez (2009), García (2015), Beghadid (2013), entre otros.

\section{MARCO COMÚN EUROPEO DE REFERENCIA: LOS NIVELES PARA EL MANEJO DE UNA LENGUA EXTRANJERA}

Según el Ministerio de Educación, cultura y deporte de España existen parámetros de manejo de una lengua extranjera, las cuales se explican en el muy conocido texto Marco Común Europeo de Referencia para las Lenguas: Aprendizaje, Enseñanza, Evaluación (2002), dichos niveles son A1, A2, B1, B2, C1 y C2. 
En este sentido, los niveles de A1 y A2 se refieren a un manejo inexperto, inicial y básico en los cuales el hablante puede:

participar en una conversación de forma sencilla siempre que la otra persona esté dispuesta a repetir lo que ha dicho o a decirlo con otras palabras y a una velocidad más lenta, y me ayude a formular lo que intento decir. Planteo y contesto a preguntas sencillas sobre temas de necesidad inmediata o asuntos muy habituales. (Marco Común Europeo de Referencia, 2002, pág. 29)

En cuanto al nivel B1 y B2 se refieren a un manejo intermedio de la lengua extranjera, el hablante es capaz de desenvolverse en interacciones más complejas, pero sin llegar a tener un novel realmente de experto.

Sé desenvolverme en casi todas las situaciones que se me presentan cuando viajo donde se habla esa lengua. Puedo participar $\begin{array}{llll}2 & 3 & 5\end{array}$ espontáneamente en una conversación que trate temas cotidianos de interés personal o que sean pertinentes para la vida diaria (por ejemplo, familia, aficiones, trabajo, viajes y acontecimientos actuales). (Marco Común Europeo de Referencia, 2002, pág. 30)

Por su parte el nivel C1 y C2 es considerado el escalón más en el manejo de una lengua extranjera, a la altura de un hablante nativo prácticamente. Esto se evidencia en el siguiente fragmento:

Me expreso con fluidez y espontaneidad sin tener que buscar de forma muy evidente las expresiones adecuadas. Utilizo el lenguaje con flexibilidad y eficacia para fines sociales y profesionales. Formulo ideas y opiniones con precisión y relaciono mis intervenciones hábilmente con las de otros hablantes. (Marco Común Europeo de Referencia, 2002, pág. 30)
En este sentido, a los estudiantes se les hizo las siguientes preguntas que respondieron así:

Según el Marco Común Europeo de Referencia ¿los niveles de manejo y conocimiento de una lengua extranjera están clasificados en cuales niveles? Y esta fue la respuesta:

- $\mathrm{A} 1, \mathrm{~A} 2, \mathrm{~B} 1, \mathrm{~B} 2, \mathrm{C} 1, \mathrm{C} 2(100 \%)$

- $\mathrm{A} 1, \mathrm{~B} 1,, \mathrm{C} 1, \mathrm{C} 2(0 \%)$

- $-1,1,2,+2,3,+3(0 \%)$

- Ninguna de las anteriores $(0 \%)$

Según el Marco Común Europeo de Referencia los niveles de manejo A1 y A2 sugieren que el manejo de la lengua extranjera es:

- Básico (100\%)

- $\quad$ Medio (0\%)

- Experto $(0 \%)$

- Ninguna de las anteriores $(0 \%)$

Según el Marco Común Europeo de Referencia los niveles de manejo B1 y B2 sugieren que el manejo de la lengua extranjera es:

- Básico (0\%)

- $\operatorname{Medio}(100 \%)$

- Experto $(0 \%)$

- Ninguna de las anteriores $(0 \%)$ 
Según el Marco Común Europeo de Referencia los niveles de manejo $\mathrm{C} 1$ y $\mathrm{C} 2$ sugieren que el manejo de la lengua extranjera es:

- Básico $(0 \%)$

- $\quad$ Medio $(0 \%)$

- Experto (100\%)

- Ninguna de las anteriores $(0 \%)$

Es evidente que los estudiantes están familiarizados con los distintos niveles de manejo de una lengua según los parámetros del Marco Común Europeo, lo que permite afirmar que este contenido de la asignatura alcanzó su objetivo de aprendizaje. Por lo menos en cuanto al manejo de la clasificación de los niveles del manejo de una lengua extranjera.

\section{TÉCNICAS PARA EL DESARROLLO DE DESTREZAS COMUNICATIVAS EN LENGUAS EXTRANJERAS}

Con respecto a la variedad de técnicas y herramientas para fomentar el desarrollo de las destrezas básicas en lengua extranjera como leer, escribir, hablar y escuchar, autores como el Ministerio de Educación de Colombia (2005) explica lo siguiente:

para enseñar una segunda lengua, es necesario partir del análisis de las necesidades comunicativas de los educandos, así como de las expectativas que los estudiantes traen al aula con respecto a la segunda lengua. De ahí que para iniciar un proceso de selección es necesario establecer los contenidos lingüísticos que se le darán al estudiante. Luego se tendrá que determinar la graduación y secuencia en la que los contenidos serán impartidos, para finalmente preocuparse por la metodología y los procedimientos más adecuados para este fin

En otras palabras, antes de iniciar el proceso de enseñanza- aprendizaje de una lengua los docentes deben evaluar las necesidades e intereses de los estudiantes. Igualmente, diseñar un plan de estudios que les permita desarrollar un aprendizaje integral de la lengua extranjera que además de estar en sintonía con el plan académico oficial también debe tener estrategias, técnicas y herramientas que se adapten al contexto social de los estudiantes. Es importante recalcar que no solo el Ministerio de Educación colombiano apoya esta premisa, también lo hacen autores como Sánchez (2014), Molina (2016), Gil (2019b), Beghadid (2013), Cortés (2000), García (2015), entre otros.

En este sentido, dentro del curso del que se desprende este trabajo, se promueve el uso de distintas técnicas y herramientas para el desarrollo de las destrezas comunicativas en lengua extranjera. Tales como la lectura reflexiva, la exposición oral, la mesa redonda, la elaboración de mapas mentales, la dramatización de obras breves, entre otras. Asimismo, a los estudiantes de este curso se les hizo las siguientes preguntas relacionadas con este punto y esto fue lo que respondieron:

Las destrezas que más deben desarrollarse en una clase de enseñanza de lengua extranjera son:

- $\quad$ Comprensión y producción de textos escritos $(0 \%)$

- $\quad$ Expresión y comprensión oral (0\%)

- Hablar y escuchar (2\%)

- $\quad$ Todas las anteriores $(98 \%)$

- $\quad$ Ninguna de las anteriores

Las destrezas más importantes que deben desarrollar los estudiantes de lenguas extranjeras son:

- Comprensión lectora y Expresión escrita $(0 \%)$ 
- $\quad$ Comprensión auditiva, Interacción oral y Expresión escrita (12\%)

- $\quad$ Comprensión auditiva, Comprensión lectora, Interacción oral, Expresión oral Expresión escrita (88\%)

- $\quad$ Ninguna de las anteriores

Es evidente que los estudiantes están familiarizados con la importancia de promover el desarrollo de las 4 destrezas básicas en la enseñanza - aprendizaje de una lengua extranjera. Y este resultado está muy relacionado con las posturas de autores como Melero (2000), Marrero (2006), Martínez (2009), Gil (2019) entre otros, que plantean que dentro del proceso del aprendizaje de una segunda lengua las destrezas que desarrolle el estudiante serán las verdaderas protagonistas.

\section{CONCLUSIONES GENERALES}

A lo largo de este trabajo, se ha determinado que 237 la encuesta aplicada a los estudiantes, si bien solo es un primer acercamiento y se considera que se requiere aplicar otros instrumentos para llegar a conclusiones más generalizables, ha permitido revelar lo siguiente:

Los estudiantes futuros docentes de lenguas, conocen la importancia de emplear varios enfoques y métodos para la enseñanza del español como lengua extranjera lo que es respaldado por los aportes teóricos de autores Martí (2017), Cortés (2000), García82015), entre otros. Que han señalado que los docentes de lenguas extranjeras deben basar su praxis en la variedad de técnicas, estrategias y herramientas que los diferentes enfoques ofrecen. En otras palabras, un solo enfoque no cubriría todas las necesidades e intereses de los estudiantes en su proceso de aprendizaje de la segunda lengua.

Asimismo, los estudiantes si bien conocen los niveles de manejo de una segunda lengua según el Marco Común Europeo todavía no muestran una mayor experticia en los mismos, probablemente debido a que aún están en formación. Sin embargo, se cree que el hecho de que conozcan e identifiquen estos niveles es una clara señal de que han alcanzado los objetivos de aprendizaje fijados por el curso electivo.

Para finalizar, se cree que esta materia electiva todavía tiene múltiples matices por enfocar y que en este primer acercamiento ha mostrado que los estudiantes de lenguas extranjeras pese a que no han culminado sus estudios, ya tienen una visión pragmática de la praxis del docente de lenguas. Sin duda alguna, este es un ítem sumamente atractivo para iniciar más adelante una investigación más detallada y rigurosa.

\section{REFERENCIAS BIBLIOGRÁFICAS}

Beghadid, H. M. (2013). El enfoque comunicativo, una mejor guía para la práctica docente - Argelia: ELE e Interculturalidad. Recuperado de http://cvc.cervantes. es/ensenanza/biblioteca ele/ publicaciones centros/PDF/oran 2013 116 beghadid.pdf

Casero, E. (2016) La importancia de las tic para la enseñanza de idiomas en el aula multicultural (Tesis doctoral) Universidad Nacional de Educación a Distancia. Madrid

Cervantes, D. (2019). Las redes sociales y el aprendizaje de la lengua extranjera. Revista Boletín Redipe, 8(11), 117-123. https://doi.org/10.36260/rbr.v8i11.855

Cortés Moreno, M. (2000). Guía para el profesor de idiomas: didáctica del español y segundas lenguas. Barcelona: Octaedro.

García, E. (2015). Visión Práctica del Enfoque Comunicativo de la Lengua . España: Universidad de Alicante. Recuperado 
de $\quad 50 \quad$ https://rua.ua.es/dspace/ bitstream/10045/47686/1/Vision practica_del_enfoque_comun icativo de_la_lengua_GARCIA_LOPEZ_ ELENA.pdf.

Gil Olivera, N. A. . (2019). El enfoque por tareas en la enseñanza de lenguas extranjeras: reflexiones de su origen y relación con otros enfoques. Revista Boletín Redipe, 8(9), 170-181. https://doi.org/10.36260/ rbr.v8i9.820

Gil Olivera, N. A. . (2019b). Ambiente virtual de aprendizaje: beneficios y ventajas para enseñanza del francés como L2. Revista Boletín Redipe, 8(11), 91-99. https://doi. org/10.36260/rbr.v8i11.852

González, D, Ladino, J. y Escobar. D. (2020) Percepciones de estudiantes sobre los métodos de enseñanza en los cursos de Inglés de una licenciatura en lenguas extranjeras de una universidad colombiana: Un estudio de caso. Revista Espacios, 41 (48). http://www.revistaespacios.com/ a20v41n48/20414803.html

Marco común europeo de referencias para las lenguas y culturas (2002). España: Ministerio de cultura y deportes

Martí, J. (2017) El español como lengua extranjera. Sapientia, 126. DOI: http:// dx.doi.org/10.6035/Sapientia126

Marrero, B. (2006). Hacía el desarrollo de la habilidad de la expresión escrita y sus implicaciones didácticas en el proceso de adquisición del idioma español como segunda lengua. Granada : Universidad de Granada .

Martínez, M. (2009). Enfoque comunicativo y la enseñanza de lenguas extranjeras. Cuba: Centro virtual Cervantes.
Melero, P. (2000). Métodos y enfoques en la enseñanza/aprendizaje del español como lengua extranjera. Madrid: Edelsa

Mendoza, A. (2003). Didáctica de la Lengua y la Literatura .España: Pearson Education

Molina, M. (2016) El aprendizaje por tareas en el aula de lenguas extranjeras. Tesis de grado en Educación primaria (Tesis de pregrado) Universidad de Cantabria, España.

Molina, S. \& Ferreira, J. (2008). Explotación didáctica de textos literarios en la enseñanza del español como lengua extranjera. En A. Clavero \& $\mathrm{M}$. Fernández (eds.), El profesor de español LE/L2 Actas del XIX Congreso Internacional de la Asociación para la Enseñanza del Español como Lengua Extranjera (ASELE), (p. 675). Cáceres: Universidad de Extremadura.

Pastran Chirinos, M., Cervantes Cerra, D., Ahumada Ebratt, D. D., \& Gil Olivera, N. A. (2020). Reflexiones sobre el uso del smartphone en el aula de lenguas extranjeras. Revista Boletín Redipe, 9(12), 265-272. https://doi. org/10.36260/rbr.v9i12.1151

Pinto, E. C., y Vargas, F. (2015). Percepciones sobre el proceso de enseñanza aprendizaje del inglés de los estudiantes y docentes de la universidad piloto de Colombia, seccional del Alto Magdalena. (Tesis de Maestría). Universidad del Tolima. Ibagué, Colombia.

Rall, D. (2015). Por una ampliación del concepto de "enfoque comunicativo". México: C.E.L.E. UNAM. http://ela. cele.unam.mx./index.php/ela/article/ download/93/80 
Rojas Moreno, W. E. (2019). La cultura popular para la enseñanza de español como lengua extranjera. Cuadernos De Lingüística Hispánica, (34). https://doi. org/10.19053/0121053X.n34.2019.9373

Sánchez, S. (2014) Los efectos de las estrategias de retroalimentación oral en la adquisición de segundas lenguas. Colindancias, 5, 283-311. Recuperado de https://dialnet.unirioja.es/descarga/ articulo/5249381.pd

Zayas, F. (2014). Problemas metodológicos en la enseñanza de lenguas extranjeras: ¿son las TIC una solución? Rastros Rostros, 16(30). 